G. Celi ${ }^{1}$, I. Brusca ${ }^{2}$, E. Scala ${ }^{3}$, D. Villalta ${ }^{4}$, E. Pastorello 5 , L. Farioli ${ }^{6}$, G. Cortellini ${ }^{7}$, G. Deleonardi ${ }^{8}$, P. Galati ${ }^{8}$, L. Losappio ${ }^{5}$, G. Manzotti ${ }^{9}$, B. Pirovano ${ }^{10}$, L. Muratore $^{11}$, F. Murzilli ${ }^{12}$, F. Cucinelli ${ }^{12}$, A. Musarra ${ }^{13}$, M. Cilia ${ }^{13}$, E. Nucera ${ }^{14}$, A. Aruanno ${ }^{14}$, F. Ria ${ }^{15}$, M.F. Patria ${ }^{16}$, E. Varin ${ }^{16}$, B.R. Polillo ${ }^{17}$, V. Sargentini ${ }^{18}$, O. Quercia ${ }^{19}$, C.G. Uasuf ${ }^{20}$, S. Zampogna ${ }^{21}$, M. Carollo ${ }^{22}$, S. Graci ${ }^{23}$, R. Asero ${ }^{1}$

\title{
House dust mite allergy and shrimp allergy: a complex interaction
}

\author{
${ }^{1}$ Allergology Clinic, San Carlo Clinic, Paderno Dugnano (MI), Italy \\ ${ }^{2}$ UOC of Clinical Pathology, Buccheri La Ferla F.B.F. Hospital, Palermo, Italy \\ ${ }^{3}$ Allergy Unit, Immacolata Dermopathic Institute, IDI-IRCCS, Rome, Italy \\ ${ }^{4}$ SSD of Immunology and Allergology, S. Maria degli Angeli Hospital, Pordenone, Italy \\ ${ }^{5}$ Complex Structure of Allergology and Immunology, ASST GOM Niguarda, Milan, Italy \\ ${ }^{6}$ Department of Laboratory Medicine, ASST GOM Niguarda, Milan, Italy \\ ${ }^{7}$ Operative Unit of Internal Medicine Rimini, Allergology Clinic, Azienda Sanitaria Romagna, Rimini, Italy \\ ${ }^{8}$ LUM AUSL, Bologna, Italy \\ ${ }^{9}$ Allergology Service, Beato Palazzolo Nursing Home, Bergamo, Italy \\ ${ }^{10}$ Laboratory Medicine Service, ASST Bergamo Ovest, Bergamo, Italy \\ ${ }^{11}$ UOC of Allergology and Immnology, Lecce POV Fazzi ASL Clinic, Lecce, Italy \\ ${ }^{12}$ UOSD of Allergology, S.S. Filippo e Nicola Hospital, Avezzano (AQ), Italy \\ ${ }^{13}$ Allergology Service, Scilla’s Nursing Home, Scilla (RC), Italy \\ ${ }^{14}$ Allergology Service, University Hospital A. Gemelli Foundation, Rome, Italy \\ ${ }^{15}$ General Pathology Institute, University Hospital A. Gemelli Foundation, Rome, Italy \\ ${ }^{16}$ IRCCS Ca' Granda Foundation, Maggiore Policlinico Hospital, Pediatric Area, Milan, Italy \\ ${ }^{17}$ Allergology Service, UOC Internal Medicine, S. Spirito and Nuovo Regina Margherita Hospital Center, Rome, Italy \\ ${ }^{18}$ S Laboratory Allergology Service, UOC Clinical Pathology, S. Filippo Neri Hospital, Rome, Italy \\ ${ }^{19}$ Allergology Unit, Internal Medicine, Faenza Hospital, Faenza (RA), Italy \\ ${ }^{20}$ Allergic Diseases Center Bonsignori, Biomedicine and Molecular Immunology Institute, CNR, Palermo, Italy \\ ${ }^{21}$ Pediatric Emergency Room, Ciaccio Apulian Hospital Company, Catanzaro, Italy \\ ${ }^{22}$ Pathology and Biochemistry Clinic, Magna Graecia University, Catanzaro, Italy \\ ${ }^{23}$ A. Mirri Experimental Zooprophylactic Sicily's Institute, Palermo, Italy
}

\section{KEY WORDS}

Food allergy; peach allergy; lipid transfer protein; SPT; diagnosis.

\section{Corresponding author:}

Riccardo Asero

Ambulatorio di Allergologia, Clinica San Carlo

Ospedale Street 21

20037 Paderno Dugnano (MI), Italy

E-mail: r.asero@libero.it

Doi

10.23822/EurAnnACI.1764-1489.108

(c) 2020 Associazione Allergologi Immunologi Italiani Territoriali e Ospedalieri - AAllTO. Published by EDRA SpA. All rights reserved. 


\section{Summary}

Background and objective. Sensitization and allergy to shrimp among Italian house dust mite allergic patients are not well defined and were investigated in a large multicenter study. Methods. Shrimp sensitization and allergy were assessed in 526 house dust mite (HD$M$ )-allergic patients submitted to the detection of IgE to Der $p 10$ and 100 atopic controls not sensitized to HDM. Results. Shrimp allergy occurred in $9 \%$ of patients (vs $0 \%$ of 100 atopic controls not sensitized to HDM; $p<0.001$ ). Shrimp-allergic patients were less frequently hypersensitive to airborne allergens other than HDM than crustacean-tolerant subjects (35\% vs 58.8\%; $p<0.005)$. Only $51 \%$ of tropomyosin-sensitized patients had shrimp allergy, and these showed significantly higher Der $p 10$ IgE levels than shrimp-tolerant ones (mean $22.2 \mathrm{KU} / \mathrm{l}$ vs $6.2 \mathrm{KU} / \mathrm{l} ; \mathrm{p}<0.05)$. Altogether $53 \%$ of shrimp-allergic patients did not react against tropomyosin. Conclusions. Shrimp allergy seems to occur uniquely in association with hypersensitivity to HDM allergens and tropomyosin is the main shrimp allergen but not a major one, at least in Italy. Along with tropomyosin-specific IgE levels, monosensitization to HDM seems to represent a risk factor for the development of shrimp allergy among HDM allergic patients.

\section{Introduction}

House dust mites are one of the main causes of respiratory allergy worldwide, and shrimp represents the second cause of primary food allergy in Italy (1). These two allergies are strictly interconnected as both mites and shrimps are invertebrates and share cross-reacting allergens, the best known being tropomyosin (table I). Shrimp allergens identified so far belong to diverse protein families characterized by conserved three-dimensional structures leading to potential IgE cross-reactivity among different members of crustaceans and mollusks (2). It is presently still unclear whether, in patients allergic to both house dust mite and crustaceans, sensitization occurs via the respiratory or the gastrointestinal tract. Prevalence studies of shrimp allergy in house dust mite allergic patients are missing. In the present work we investigated a large population of house dust mite-allergic patients, the vast majority selected within a national multicenter study (3) with the aim to detect the prevalence and features of shrimp allergy.

Figure 1 - Venn diagram showing the prevalence and serological features of shrimp allergy among 526 HDM-allergic patients.

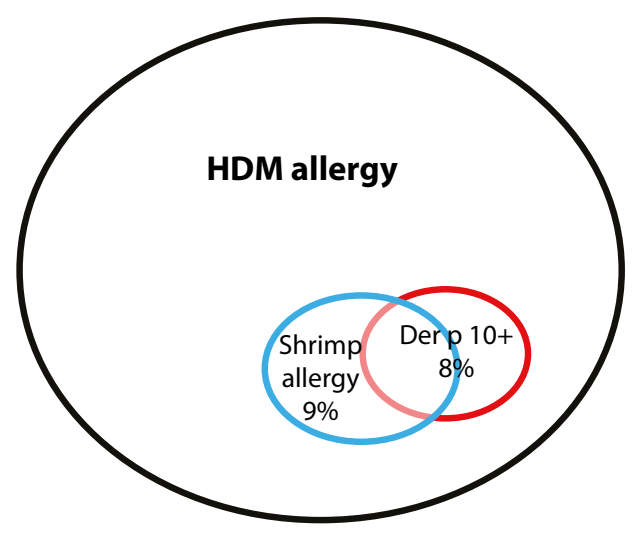

\section{Materials and methods}

\section{Patients}

Five hundred and twenty-six house dust mite-allergic patients (M/F: 261/265; mean age 28.2 years, range $4-79$ years) were studied. This population was virtually the same recently investigated to study the clinical significance of Der p 23, a major HDM allergen (3). Methods employed to diagnose HDM allergy included a positive SPT with a commercial extract of either Dermatophagoides pteronyssinus (D1) or Dermatophagoides farinae (D2), and the measurement of $\mathrm{IgE}$ specific for the HDM whole extracts D1, and D2, by ImmunoCAP (ThermoFisher Scientific, Uppsala, Sweden). IgE specific for Der p 10, the house dust mite tropomyosin, were measured as well in all study patients. Levels exceeding $0.35 \mathrm{kU} / \mathrm{L}$ were considered positive; this cut-off level was chosen with the aim to improve the specificity of in-vitro tests. Further, all patients underwent SPT with a large series of commercial extracts of seasonal (grass, mugwort, ragweed, pellitory, plantain, birch, olive, and cypress) and perennial (Alternaria, cat and dog dander) allergens. Patients were thoroughly interviewed about their tolerance to crustaceans. Those reporting suspect allergic reactions associated with the ingestion of shrimp or other invertebrates (i.e., oral allergy syndrome, contact urticaria, generalized urticaria, asthma, or anaphylaxis) underwent SPT with either commercial extract of shrimp (1:20 w/v; ALK-Abello', Madrid Spain) or fresh shrimp and/or shrimp-specific IgE measurement to confirm sensitization status. Skin tests with fresh material were carried out using the most common seawater shrimp species eaten in Italy, all belonging to the Penaeideae family (Aristeus antennatus, Parapenaeus longirostris, Parapeneopsis cornuta and Melicertus kerathurum). Patients scoring positive on SPT and/or on ImmunoCAP were considered as clinically allergic to shrimp.

One hundred randomly selected atopic patients sensitized to different airborne allergens except house dust mites were assessed for crustacean allergy in the same way and were used as controls. 
Table I - House dust mite allergens. Offcial Shared allergens between house dust mite and shrimp are highlighted.

Allergen

Biochemical name

MW Allergen

\section{Dermatophagoides farinae}

\begin{tabular}{|c|c|c|c|}
\hline & & & \\
\hline Der f 1 & Cysteine protease & 27 & Der p 1 \\
\hline Der $\mathrm{f} 2$ & NPC2 family & 15 & Der p 2 \\
\hline Der $\mathrm{f} 3$ & Trypsin & 29 & Der p 3 \\
\hline \multirow[t]{2}{*}{ Der f 4} & alpha-amylase & 58 & Der $\mathrm{p} 4$ \\
\hline & & & Der $\mathbf{p} 5$ \\
\hline Der $\mathrm{f} 6$ & Chymotrypsin & 25 & Der p 6 \\
\hline Der f 7 & Bactericidal permeability-increasing like protein & 30 & Der p 7 \\
\hline \multirow[t]{2}{*}{ Der $\mathrm{f} 8$} & Glutathione S-transferase & 32 & Der p 8 \\
\hline & Collagenolytic serine protease & 29 & Der $p 9$ \\
\hline Der $f 10$ & Tropomyosin & 37 & Der $\mathbf{p} 10$ \\
\hline Der f 11 & Paramyosin & 98 & Der p 11 \\
\hline Der $f 13$ & Fatty acid binding protein & & Der p 13 \\
\hline Der $f 14$ & Apolipophorin & 177 & Der p 14 \\
\hline Der f 15 & Chitinase & 98 & Der p 15 \\
\hline Der f 16 & Gelsolin/villin & 53 & \\
\hline Der f 17 & Calcium binding protein & 53 & \\
\hline Der $\mathrm{f} 18$ & Chitin-binding protein & 60 & Der p 18 \\
\hline Der $\mathrm{f} 20$ & Arginine kinase & 40 & Der p 20 \\
\hline Der f 21 & & 14 & Der p 21 \\
\hline \multicolumn{4}{|l|}{ Der $\mathrm{f} 22$} \\
\hline Der f 23 & Peritrophin-like protein & 19 & Der p 23 \\
\hline Der $f 24$ & $\begin{array}{l}\text { Ubiquinol-cytochrome c reductase } \\
\text { binding protein homologue }\end{array}$ & 13 & Der p 24 \\
\hline Der $f 25$ & Triosephosphate isomerase & 34 & \\
\hline Der $\mathrm{f} 26$ & Myosin alkali light chain & 18 & \\
\hline Der f 27 & Serpin & 48 & \\
\hline Der $f 28$ & Heat Shock Protein & 70 & \\
\hline Der f 29 & Peptidyl-prolyl cis-trans isomerase (cyclophilin) & 16 & \\
\hline Der $\mathrm{f} 30$ & Ferritin & 16 & \\
\hline Der f 31 & Cofilin & 15 & \\
\hline Der $f 32$ & Secreted inorganic pyrophosphatase & 35 & \\
\hline Der f 33 & alpha-tubulin & 52 & \\
\hline Der f 34 & enamine/imine deaminase & 16 & \\
\hline Der $f 35$ & & 14 & \\
\hline \multirow[t]{2}{*}{ Der $\mathrm{f} 36$} & & 23 & Der p 36 \\
\hline & Petrotrophic like protein domain & 30 & Der p 37 \\
\hline
\end{tabular}




\section{Statistics}

Statistical methods as well as ethical issues have been detailed elsewhere (3). Probability levels $<5 \%$ were considered statistically significant.

\section{Ethical issues}

The clinical part of the study as well as specific IgE measurement were carried out as part of the clinical routine of every participating center. Patients gave an informed consent to the use of their clinical data in an anonymous form. The study was approved by the internal review board of the leading center. In view of the essentially observational nature of the study a formal approval by an external ethical committee was not requested.

\section{Results}

The main findings are summarized in figure $\mathbf{1}$. The prevalence of shrimp allergy in the general house dust mite allergic population was $45 / 526(9 \%)$ vs $0 / 100(0 \%)$ in the control population ( $\mathrm{p}<$ $0.001)$. No differences in the prevalence of shrimp allergy between female $(7.5 \%)$ and male $(9.6 \%)$ patients was detected. Similarly, patients allergic and not allergic to crustaceans showed the same mean age (30 [16.2] years vs 28.2 [16.2] years, respectively), and no difference in the prevalence of asthma was observed between patients allergic or tolerant to shrimp ( $40 \%$ vs $40 \%$, respectively). In contrast, patients with crustacean allergy were much less frequently hypersensitive to airborne allergens other than house dust mites than tolerant patients ( $35 \%$ vs $58.8 \%$; $\mathrm{p}<0.005$ ).

The prevalence of hypersensitivity to tropomyosin in the study population was $7.8 \%(41 / 526)$. Of tropomyosin reactors, only $21(51 \%)$ were clinically allergic to crustaceans, whereas 20 (49\%) reported good tolerance to shrimp and other invertebrates. Interestingly, those with shrimp allergy showed a significantly higher mean level of IgE to Der p 10 than patients reporting good tolerance to crustaceans (22.2 [SD 28.0] KU/1 vs 6.2 [9.6] KU/l; p < 0.05). Altogether, Der p 10 reactors were more frequently allergic to crustaceans than patients that did not show IgE specific for Der p 10 (21/41 [51\%] vs 24/485 [4.9\%]; $\mathrm{p}<0.001)$. Nonetheless, notably $24 / 45$ (53\%) patients allergic to crustaceans did not react against tropomyosin. Finally, no difference in the prevalence of shrimp allergy was detected between patients monosensitized to Der p 10 (7/14 [50\%]) and Der $\mathrm{p} 10$ reactors who were sensitized to other mite allergens also (13/27 [48\%]; p: NS).

\section{Discussion}

The present study, which was carried out on a large population of patients with clinically defined house dust mite allergy, shows once more to which extent hypersensitivity to house dust mites and to shrimp are strictly linked. In effect, none among the atopic controls reported symptoms suggestive of shrimp allergy whereas the prevalence of shrimp allergy in the study population was nearly $10 \%$. Such prevalence suggests that the cross-reactivity between HDM and other invertebrates involves minor mite allergens. Tropomyosin was the first shrimp allergen to be identified more than 25 years ago (4). Although it has been considered the major shrimp allergen ever since, recent multicenter studies carried out in the Mediterranean area were able to detect tropomyosin hypersensitivity in less than 50\% of shrimp allergic patients (5). This observation was fully confirmed by the present study that was carried out on a completely different population, where $53 \%$ of shrimp-allergic patients were not tropomyosin reactors. Further, interestingly, among tropomyosin-hypersensitive patients the occurrence of shrimp allergy was strongly related to specific IgE levels, suggesting the clinical relevance of sensitization degree. Nonetheless, the present study confirmed the association between tropomyosin sensitization and shrimp allergy.

A number of shrimp allergens other than tropomyosin have been detected during the last years (2); most of these seem phylogenetically conserved throughout the invertebrates' kingdom and hence able to cross react with homologous house dust mite allergens $(5,6)$. Although in-vitro cross-inhibition experiments were not carried out in the present study it has to be considered that the whole study population was represented by patients with house dust mite-induced respiratory allergy, and no atopic control reported a history of food allergy to shrimps. In one shrimp allergic patients that did not react to recombinant Der $\mathrm{p}$ 10 the relevant shrimp allergen, that showed a molecular weight at about $100 \mathrm{kDa}$ on immunoblot analysis was characterized by mass spectrometry (3) as paramyosin, a potentially cross-reacting muscular allergen of invertebrates.

Another interesting finding was the significantly higher prevalence of shrimp allergy among subjects monosensitized to HDM than among those who reacted to different airborne allergens. This observation is in keeping with similar findings in patients with food allergy to lipid transfer protein, that show more severe reactions if they are monosensitized and less severe allergic reactions in case of co-sensitization to airborne allergens (7). These findings might suggest that the dispersion of specific $\mathrm{IgE}$ reactivity over a larger number of targets is protective against severe allergic reactions or against food allergy per se. In conclusion, shrimp allergy seems to occur uniquely in association with hypersensitivity to HDM allergens and, at least in this geographical area, tropomyosin is the main shrimp allergen but not a major one. Along with tropomyosin-specific IgE levels, monosensitization to HDM seems to represent a risk factor for the development of shrimp allergy among HDM allergic patients. 


\section{Obituary}

This paper is in memory of our colleague Elena Varin.

\section{Author contribution statement}

Every author listed participated in the recruitment of patients and in the clinical workup at their own allergy centers. RA conceived and managed the multicenter study and wrote the manuscript. ES and DV revised the manuscript. GC performed the statistical analyses

\section{Conflict of interests}

The authors declare that they have no conflict of interests.

\section{References}

1. Asero R, Antonicelli L, Arena A, Bommarito L, Caruso B, et al. EpidemAAITO: features of food allergy in Italian adults attending allergy clinics: a multi-centre study. ClinExp Allergy 2009;39:547-55.
2. Matricardi PM, Kleine-Tebbe J, Hoffmann HJ, Valenta R, Hilger C, Hofmaier S, et al. EAACI Molecular Allergology User's Guide. Pediatr Allergy Immunol 2016;27 Suppl 23:1-250.

3. Celi G, Brusca I, Scala E, Villalta D, Pastorello E, Farioli L, Cortellini G, et al. House dust mite allergy in Italy-Diagnostic and clinical relevance of Der p 23 (and of minor allergens): A real-life, multicenter study. Allergy 2019;74:1787-89. doi: 10.1111/all.13776.

4. Shanti KN, Martin BM, Nagpal S, Metcalfe D DD, Rao PVS. Identification of tropomyosin as the major shrimp allergen and characterization of its IgE-binding epitopes. J Immunol 1993; 151: 5354-63.

5. Asero R, Mistrello G, Amato S, Ariano R, Colombo G, Conte ME, et al. Shrimp allergy in Italian adults: a multicenter study showing a high prevalence of sensitivity to novel high molecular weight allergens.Int Arch Allergy Immunol 2012; 157:3-10.

6. Giuffrida MG, Villalta D, Mistrello G, Amato S, Asero R. Shrimp allergy beyond Tropomyosin in Italy: clinical relevance of Arginine Kinase, Sarcoplasmic calcium binding protein and Hemocyanin. Eur Ann Allergy ClinImmunol 2014; 46:172-7.

7. Pastorello EA, Farioli L, Pravettoni V, et al. Pru p 3-sensitised Italian peach allergic patients are less likely to develop severe symptoms when also presenting $\operatorname{IgE}$ antibodies to Pru p 1 and Pru p 4. Int Arch Allergy Immunol 2011; 156:362-72. 